\title{
Early reversible leukoencephalopathy and unilateral sixth cranial nerve palsy in mild COVID-19 infection
}

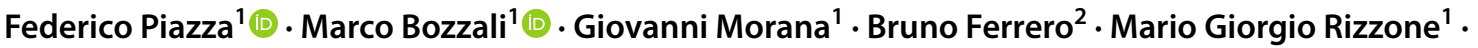 \\ Carlo Alberto Artusi ${ }^{1}$ (D) Mattia Parisi ${ }^{2}$. Alice Robert ${ }^{2}$. Gabriele Imbalzano ${ }^{2}$. Alberto Romagnolo ${ }^{1}$ (1) . \\ Maurizio Zibetti ${ }^{1}$ - Leonardo Lopiano ${ }^{1}$ (i)
}

Received: 20 April 2021 / Accepted: 31 July 2021 / Published online: 4 September 2021

(C) Fondazione Società Italiana di Neurologia 2021

\begin{abstract}
Objectives To provide new insights into neurological manifestations of COVID-19. We describe a patient with mild COVID19 associated with diplopia from right sixth cranial nerve palsy and early diffuse leukoencephalopathy, successfully treated with intravenous methylprednisolone.

Methods The patient was evaluated for diplopia that occurred 1 day after the onset of fever, myalgia, and headache. A complete neurological workup, including neurological examination, cerebrospinal fluid (CSF) analysis with viral polymerase chain reaction (PCR), serum autoimmune encephalitis, and anti-nerve antibodies and brain magnetic resonance imaging (MRI), was performed.

Results Clinical examination revealed incomplete right sixth cranial nerve palsy. Brain MRI showed diffuse confluent fluidattenuated inversion recovery (FLAIR) hyperintense white matter abnormalities, while CSF analysis showed mild hyperproteinorrachia $(61 \mathrm{mg} / \mathrm{dL})$ without pleocytosis. The patients were treated with high-dose intravenous methylprednisolone with rapid improvement of neurological symptoms and resolution of CSF and MRI abnormalities.

Discussion Our report shows that COVID-19 may predominantly present with neurological symptoms; furthermore, it argues the notion of leukoencephalopathy as a typical feature of a severe case of the disease. Mechanisms underpinning neurological symptoms in COVID-19 still need to be elucidated; nonetheless, early recognition and prompt management may ensure their improvement or even complete recovery and are therefore recommended.
\end{abstract}

Keywords COVID-19 $\cdot$ MRI $\cdot$ Cranial neuropathy $\cdot$ Leukoencephalopathy

\section{Introduction}

Various neurological conditions have been reported in association with COVID-19 [1]. We describe a patient presenting with unilateral sixth cranial nerve palsy and early reversible leukoencephalopathy, successfully treated with high-dose intravenous methylprednisolone.

Federico Piazza

federico.piazza@unito.it

1 Department of Neuroscience "Rita Levi Montalcini", University of Turin, Via Cherasco 15, 10126 Turin, Italy

2 AOU Città Della Salute E Della Scienza, Turin, Italy

\section{Case report}

A 47-year-old male was admitted to our Emergency Department in Turin, Italy, for diplopia that occurred 1 day after the onset of fever (max. $38.7^{\circ} \mathrm{C}$ ), myalgia, and headache. Past medical history was unremarkable. A first SARS-CoV-2 nasopharyngeal swab was positive. Chest $\mathrm{X}$-ray and lung ultrasound were normal. Neurological examination revealed non-fluctuating diplopia and incomplete right sixth cranial nerve palsy (mild-degree adduction of the right eye in primary position of gaze and ipsilateral deficit of abduction). Head computed tomography (CT) did not show abnormalities. Laboratory workup showed leukopenia $(2800$ cells $/ \mu \mathrm{L})$ with lymphopenia $(790$ cells $/ \mu \mathrm{L})$; $\mathrm{C}$-reactive protein and D-dimer were normal. Serum antiganglioside, anti-myelin-associated glycoprotein (MAG), paraneoplastic syndrome, and autoimmune encephalitis 
antibodies were negative. Brain 1.5-T magnetic resonance imaging (MRI) on admission demonstrated diffuse confluent bilateral fluid-attenuated inversion recovery (FLAIR) hyperintense white matter abnormalities involving the perirolandic subcortical region, centrum semiovale, and corona radiata; increased FLAIR signal was also evident along the corticospinal tracts (at the posterior limb of the internal capsule and in the pons), as well as in the middle cerebellar peduncles (Fig. 1). Cerebrospinal fluid (CSF) analysis (lumbar puncture's opening pressure $=17 \mathrm{cmH}_{2} \mathrm{O}$ ) revealed mild hyperproteinorrachia $(61 \mathrm{mg} / \mathrm{dL})$ without pleocytosis. CSF real-time (RT) PCR for SARS-CoV-2 and common pathogens was negative. After admission to a COVID-19 ward, the patient was treated with highdose $(1000 \mathrm{mg})$ intravenous methylprednisolone for 3 days with rapid improvement of diplopia. Intravenous steroid was tapered and afterwards converted to oral prednisone. A second CSF analysis performed 10 days after onset of diplopia (lumbar puncture's opening pressure $=16 \mathrm{cmH}_{2} \mathrm{O}$ ) with isoelectric focusing (IEF) showed normalized proteins and no oligoclonal bands. Brain MRI repeated on the same scanner 1 week later demonstrated complete resolution of the previously seen abnormalities (Fig. 1). The patient was discharged on oral prednisone, which was suspended 10 days later. On discharge, blood test workup returned all parameters within normal ranges. Nasopharyngeal swab became negative 20 days after discharge. A neurological examination 1 month after the onset of diplopia confirmed complete clinical recovery. Two months after discharge, the patient underwent electrodiagnostic examination (EDX) with lower limb motor and sensory conduction and blink reflex study showing no evidence of polyneuropathy. A third brain MRI performed on a 3-T scanner, 3 months after the onset of symptoms, confirmed lack of brain abnormalities (Fig. 1).

\section{Discussion}

Since the beginning of the COVID-19 outbreak, there has been great interest in possible neurological manifestations of the disease. Neurological complications had already been reported during the severe acute respiratory syndrome (SARS) and Middle East respiratory syndrome (MERS) epidemics [1]; since olfactory and gustative symptoms are very frequent in COVID-19, a particular tropism of SARS-CoV-2 for the nervous tissue is conceivable. Indeed, a direct involvement of the olfactory bulb and the cortex of gyrus rectus has been demonstrated through MRI [2]. Thus far, COVID-19 has been associated with leukoencephalopathies, acute polyneuropathies including Guillain-Barrè and Miller-Fisher syndrome, acute disseminated encephalomyelitis (ADEM), encephalitis, seizures and ischemic stroke [1], and posterior reversible encephalopathy syndrome [3-5]. In one case, viral RNA was found in the CSF of a patient with encephalitis and status epilepticus [6]. Cranial neuropathies have also been reported [7-10]. In particular, from the description of two cases with ophthalmoparesis from cranial nerve palsy whose neurological symptoms developed shortly after COVID-19 clinical onset [7] rose relevant questions about a possible direct effect of the virus on the nervous tissue [11]. Our patient showed a similar clinical course; moreover, both CSF and MRI showed early signs of central nervous system (CNS) involvement. With respect to our patient's oculomotion deficit, it is most likely to be of intra-axial origin (nucleus/root involvement), and attributable to the white matter alterations observed in the pons. A peripheral nerve involvement due to mildly increased intracranial pressure (opening pressure values approaching the upper normal limit in both lumbar puncture occasions) may also have contributed to the sixth cranial nerve palsy. An involvement of the right lateral rectus muscle seems unlikely considering the absence of abnormalities of the intra-orbital tissues on MRI and negative results on screening tests for autoimmunity.

The early reversible leukoencephalopathy we observed on MRI is of particular interest, arguing the notion that diffuse white matter involvement is a peculiar feature of COVID-19 patients with either impairment of consciousness or severe respiratory distress [12]; indeed, our patient showed neither clinical signs of encephalopathy nor severe systemic or respiratory symptoms. Viral genome was not found in the CSF, making the association with COVID-19 only probable [1]. Nonetheless, our patient had no risk factors or comorbidities which might account for acute cranial nerve palsy; moreover, it has been shown that CNS involvement in COVID-19 is more frequent among patients with reduced blood lymphocytes [13]. EDX allowed us to exclude polyneuropathy, although it could not be performed immediately due to the limitations imposed by the pandemic. In conclusion, our case suggests that COVID-19 may also involve both the peripheral and central nervous systems; moreover, CNS involvement may occur early even in the absence of severe respiratory or systemic symptoms. The mechanisms underpinning neurological symptoms in COVID-19 still need to be elucidated; nonetheless, early recognition and prompt management may ensure their improvement or even complete recovery. Careful neurological assessment and differential diagnosis are therefore mandatory also in mild cases of COVID-19. 

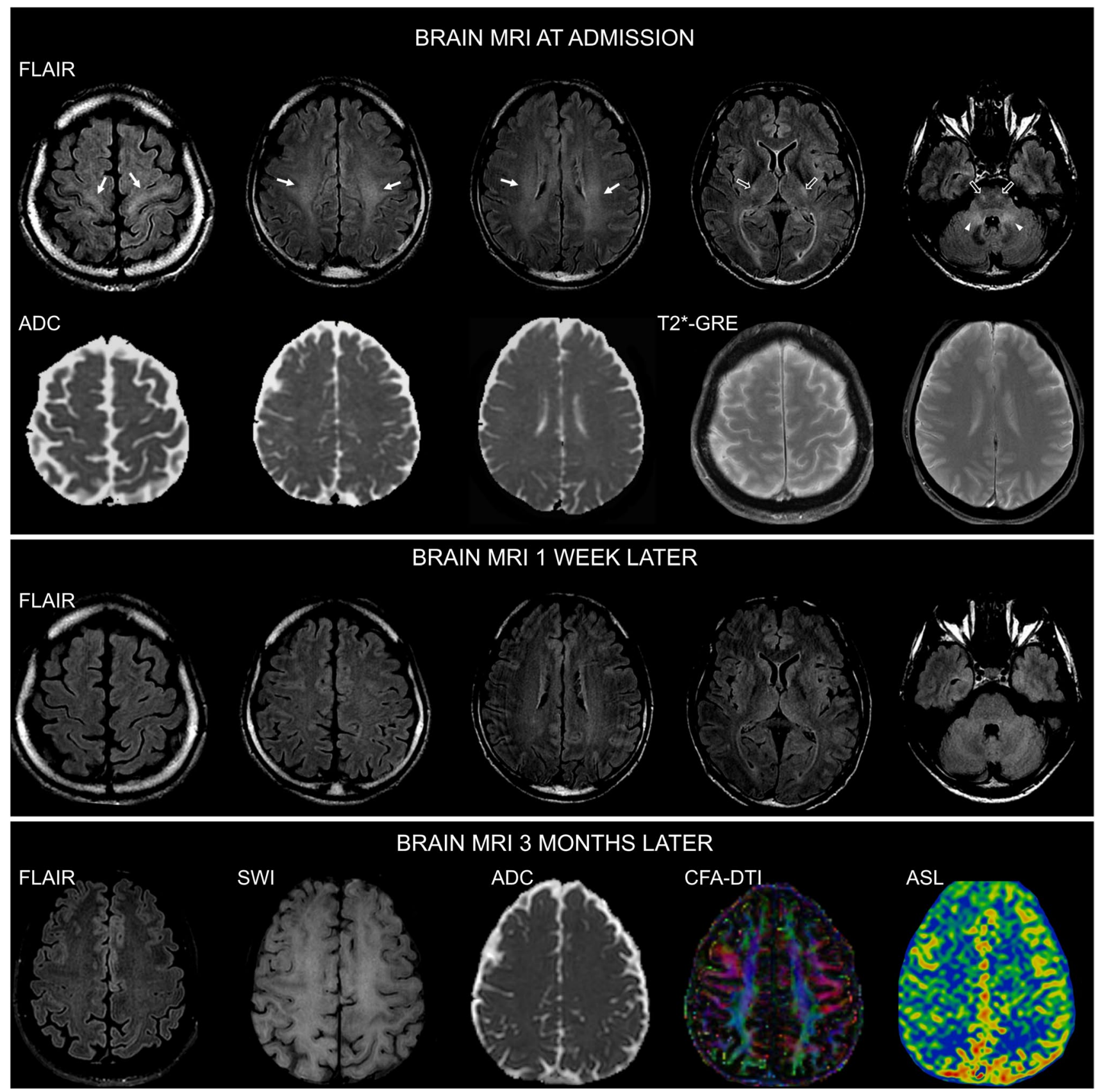

Fig. 1 Reversible leukoencephalopathy associated with COVID 19. MRI findings. Axial FLAIR images, performed at admission on a 1.5-T scanner, showed diffuse confluent bilateral white matter hyperintensity involving the perirolandic subcortical region, centrum semiovale, and corona radiata (thin arrows). There was concomitant hyperintense signal along the corticospinal tract at the posterior limb of the internal capsule and in the pons (open arrows). Increased FLAIR signal was also evident in the middle cerebellar peduncle, bilaterally (arrowheads). Apparent diffusion coefficient (ADC) and T2*-gradient echo (GRE) images did not show areas of restricted dif- fusivity, neither hemorrhagic foci. Post-contrast imaging (not shown) did not reveal pathologic enhancing areas. Axial FLAIR images performed 1 week later, on the same 1.5-T scanner, showed complete resolution of the leukoencephalopathy. Brain MRI performed 3 months later on a 3-T scanner, including high-resolution FLAIR images, susceptibility weighted imaging (SWI), ADC, colored fractional anisotropy diffusion tensor imaging (CFA-DTI), and arterial spin labeling (ASL) perfusion imaging, did not reveal any structural, microstructural, or hemodynamic brain abnormality and no long-term sequelae 
Author contribution All the authors contributed equally to the manuscript.

Data availability Not applicable.

Code availability Not applicable.

\section{Declarations}

Ethics approval Not applicable.

Consent to participate Not applicable.

Consent for publication The patient read the article and gave informed consent for publication of anonymous data in the research literature.

Conflict of interest None of the authors has conflicts of interest related to this article.

\section{References}

1. Ellul MA, Benjamin L, Singh B et al (2020) Neurological associations of COVID-19. Lancet Neurol 19(9):767-783. https://doi.org/ 10.1016/S1474-4422(20)30221-0

2. Politi LS, Salsano E, Grimaldi M (2020) Magnetic resonance imaging alteration of the brain in a patient with coronavirus disease 2019 (COVID-19) and anosmia. JAMA Neurol 77(8):10281029. https://doi.org/10.1001/jamaneurol.2020.2125

3. Princiotta Cariddi L, Tabaee Damavandi P, Carimati F et al (2020) Reversible encephalopathy syndrome (PRES) in a COVID-19 patient. J Neurol 267(11):3157-3160. https://doi.org/10.1007/ s00415-020-10001-7

4. Lallana S, Chen A, Requena M et al (2021) Posterior reversible encephalopathy syndrome (PRES) associated with COVID-19. J Clin Neurosci 88:108-112. https://doi.org/10.1016/j.jocn.2021. 03.028
5. Anand P, Lau KHV, Chung DY et al (2020) Posterior reversible encephalopathy syndrome in patients with coronavirus disease 2019: two cases and a review of the literature. J Stroke Cerebrovasc Dis 29(11):105212. https://doi.org/10.1016/j.jstrokecerebrov asdis.2020.105212

6. Moriguchi T, Harii N, Goto J et al (2020) A first case of meningitis/encephalitis associated with SARS-coronavirus-2. Int J Infect Dis 94:55-58. https://doi.org/10.1016/j.ijid.2020.03.062

7. Dinkin M, Gao V, Kahan J et al (2020) COVID-19 presenting with ophthalmoparesis from cranial nerve palsy. Neurology 95(5):221223. https://doi.org/10.1212/WNL.0000000000009700

8. Greer CE, Bhatt JM, Oliveira CA, Dinkin MJ. Isolated cranial nerve 6 palsy in 6 patients with COVID-19 infection. J Neuroophthalmol 2020;40(4):520-2. https://doi.org/10.1097/WNO.00000 00000001146. Erratum in: J Neuroophthalmol 2021;41(2):e276

9. Falcone MM, Rong AJ, Salazar H et al (2020) Acute abducens nerve palsy in a patient with the novel coronavirus disease (COVID-19). J AAPOS 24(4):216-217. https://doi.org/10.1016/j. jaapos.2020.06.001

10. Corrêa DG, Cruz LC Jr, Lopes FCR et al (2021) Magnetic resonance imaging features of COVID-19-related cranial nerve lesions. J Neurovirol 27(1):171-7. https://doi.org/10.1007/ s13365-020-00934-0

11. Dinkin M, Gao V, Kahan J et al (2020) Author response: COVID19 presenting with ophthalmoparesis from cranial nerve palsy. Neurology 95(9):411. https://doi.org/10.1212/WNL.0000000000 010411

12 Yoon BC, Buch K, Lang M et al (2020) Clinical and neuroimaging correlation in patients with COVID-19. AJNR Am J Neuroradiol 41(10):1791-6. https://doi.org/10.3174/ajnr.A6717

13. Mao L, Jin H, Wang M et al (2020) Neurologic manifestations of hospitalized patients with coronavirus disease 2019 in Wuhan. China JAMA Neurol 77(6):683-690. https://doi.org/10.1001/ jamaneurol.2020.1127

Publisher's note Springer Nature remains neutral with regard to jurisdictional claims in published maps and institutional affiliations. 